\title{
Crisis en las políticas públicas de cultura La planeación cultural del gobierno de López Obrador*
}

\author{
Crisis on the cultural public policy \\ The cultural planning of López Obrador's government
}

\author{
EDUARDO NIVÓN BOLÁN**
}

\begin{abstract}
Based on documentary analysis, this article holds that there is a public cultural policy crisis. The first sign of this crisis can be found in how objectives and goals are defined as part of the critique of the neoliberal period, in spite of similarities in practice to those established in prior years, which shows a trend where attempts to adopt these policies show discursive prevalence over practice that lacks precision. The 2020 crisis has taken the cultural sector into a difficult setting which translates into a process of de-institutionalizing cultural programs due to budgetary issues. A substantial amount of the cultural sector's issues are not unique to Mexico, thus the most relevant question is whether we are experiencing a global trend and where this process is leading public commitment to culture.
\end{abstract}

Key words: cultural planning, public policy, cultural policy, cultural public policy, cultural management

\begin{abstract}
Resumen
Basado en análisis documental, este artículo sostiene que hay una crisis en la política pública de cultura. El primer indicio de ello se encuentra en la definición de objetivos y metas como parte de la crítica al periodo neoliberal, pero en la práctica muy semejantes a los definidos en los años anteriores, lo que produce una tendencia al predominio del discurso y poca precisión en las prácticas que se intenta adoptar. La crisis de 2020 ha tomado al sector cultural en una situación difícil que se está traduciendo en un proceso de desinstitucionalización de los programas culturales debido a problemas presupuestarios. Gran parte de las dificultades que vive el sector cultural no son exclusivas de México, por ello la pregunta más relevante es si estamos viviendo una tendencia internacional y hacia dónde está llevando este proceso el compromiso público por la cultura.

Palabras clave: planeación cultural, políticas públicas, política cultural, políticas públicas de cultura, gestión cultural
\end{abstract}

\section{El desarrollo de las políticas públicas de cultura}

$\mathrm{L}$ a política cultural, un concepto amplio y elusivo, ha sido en México uno de los factores que coadyuvaron a la notable estabilidad del régimen surgido de la Revolución mexicana. En este primer momento de análisis, se entiende la política cultural como la construcción del encaje simbólico de lo político que consiguió dar sentido al nuevo Estado en cuanto un aparato legítimo de transformación de la realidad social que merecía

\footnotetext{
* Artículo recibido el 10/04/20 y aceptado el 20/05/20.

** Universidad Autónoma Metropolitana, Unidad Iztapalapa, Departamento de Antropología. Av. San Rafael Atlixco núm. 186, col. Vicentina, alcaldía Iztapalapa, 09340 Ciudad de México <nivon@xanum.uam.mx>.
} 
apoyo y adhesión de la mayoría de los ciudadanos, especialmente los trabajadores del campo y la ciudad. Maestros, artistas e intelectuales afines lograron articular un proyecto que dio valor a la cultura popular, al patrimonio y a la educación nacionalista. Se trató de un proceso cultural que contribuyó a la adhesión de los sectores populares a una organización política corporativa, conocida desde 1946 como Partido Revolucionario Institucional (PRI), y al régimen del nacionalismo revolucionario.

Esa impetuosa política cultural, cuyo empuje alcanzó hasta los años setenta, comenzó a declinar en el último tercio del siglo xx, cuando la capacidad del Estado para atender las demandas sociales se fue agotando, lo cual lo obligó a reestructurar tanto sus políticas económicas como el propio modelo de gestión pública. Ahora bien, conforme el Estado mexicano veía crecer sus dificultades de desarrollo debido a la autocracia presidencial, a la improvisación de sus políticas, a la imposición de criterios de modernización externos (al modo del Consenso de Washington) y al crecimiento de la propia oposición democrática en México, el acuerdo de desarrollar políticas públicas racionales y objetivas fue ganando terreno de legitimidad en el conjunto del Estado.

La planeación pública data de los mismos orígenes del Estado posrevolucionario, ${ }^{1}$ pero fue en los años ochenta cuando se hizo una profunda transformación normativa en este terreno. Miguel de la Madrid Hurtado, presidente de 1982 a 1988, provenía de la Secretaría de Programación y Presupuesto y había sido testigo de los excesos autocráticos de los presidentes anteriores y del derrumbe económico de 1982. En ese momento, a cargo de un Estado con graves limitaciones económicas, en un entorno internacional que impulsaba el cambio de rumbo hacia el neoliberalismo y con la presión de movimientos sociales cada vez más amplios en favor de la democracia, los derechos humanos y la defensa de las condiciones de vida amenazadas por la crisis, asumió el poder con dos objetivos básicos: una convocatoria a una renovación moral de la sociedad y la promoción de una gestión ordenada de la actividad pública a partir de la planeación estatal. Fue él también quien dio un giro radical al modelo económico imperante al terminar con el proteccionismo del Estado y abrir la economía a la competitividad internacional. Este giro administrativo coincide en nuestro país con el despliegue del paradigma de las políticas públicas recién incorporado al espacio académico en esos años ${ }^{2}$ y supuso un viraje incuestionable en la definición de la acción de gobierno. En la academia, poco a poco fue ganando interés el estudio de los procesos "de decisión y puesta en práctica de las políticas” (Aguilar Villanueva, 1992: 15).

¿Cómo ha repercutido el enfoque de las políticas públicas en el campo de la cultura? Casi al término de su periodo de gobierno, y estando por inaugurarse la administración de Carlos Salinas de Gortari, las dos cámaras legislativas constituyeron sendas comisiones de cultura, que permitieron por primera vez una atención enfocada expresamente en ese sector al margen de la educación. A los pocos días de asumir el poder, Carlos Salinas creó el Consejo Nacional para la Cultura y las Artes, Conaculta, desligando al sector cultural de la tutela inmediata de la Secretaría de Educación Pública (SEP), aunque esto iba en realidad a depender de la voluntad del titular de esta dependencia. ${ }^{3}$ De esta manera se abrió la posibilidad administrativa de planear la cultura de modo específico más allá de los lineamientos de la SEP. En efecto, hasta antes de 1988 la planeación cultural, e incluso la política cultural, estaba centrada en lo que los dos grandes institutos nacionales de cultura, el de Antropología e Historia y el de Bellas Artes y Literatura, definían para sus respectivos campos de competencia. A partir de entonces se pudo plantar una bandera en la planeación del gobierno federal y definir un presupuesto preciso para la cultura.

Esto último mueve a pensar en un cierto paralelismo con el desarrollo de la política cultural francesa, pues la primera tarea que se impuso el Ministro de Asuntos Culturales, André Malraux, fue incorporar el sector cultural a la planeación cultural (y a la necesidad de asignarle recursos). Cuando, en 1970, la Organización de las Naciones Unidas para la Educación, la Ciencia y la Cultura (Unesco) publicó el reporte Some aspects of French cultural policy, elaborado por el Departamento de Estudios e Investigación del Ministerio de Cultura de Francia, ${ }^{4}$ los autores iniciaron el documento señalando precisamente el vuelco pro-

\footnotetext{
1 "Los antecedentes de la planificación en el México contemporáneo, se remontan a los años posteriores al movimiento revolucionario de 1917 y su inicio coincide con la primera transmisión pacífica del poder" (Ruiz Dueñas, 1983: 35).

2 La primera maestría en esta materia surgió en el Instituto Tecnológico Autónomo de México en 1987 (Aguilar Villanueva, 1992: 11)

3 Desde 1959 hasta 1988 existió una Subsecretaría de Cultura con objetivos institucionales muy diversos.

4 http://unesdoc.unesco.org/images/0000/000012/001205eo.pdf.
} 
ducido por la planificación, uno de los elementos que luego fueron considerados dentro de los estándares mínimos de actuación de cualquier instancia cultural. ${ }^{5}$

\section{La normatividad federal sobre planeación}

Es conveniente tener en cuenta al menos tres elementos normativos de la planeación gubernamental en México según la Ley de Planeación:

a) Sentido general: La ley establece que la planeación es un medio para "atender el eficaz desempeño de la responsabilidad del Estado sobre el desarrollo equitativo, incluyente, integral, sustentable y sostenible del país, con perspectiva de interculturalidad y de género, y deberá tender a la consecución de los fines y objetivos políticos, sociales, culturales, ambientales y económicos" (art. 2).

b) Se trata de un instrumento de gobernanza. Tanto el plan nacional como los sectoriales e institucionales son el marco de referencia para "dar cuenta anualmente al Congreso de la Unión del estado que guardan sus respectivos ramos, [e] informarán del avance y grado de cumplimiento de los objetivos y prioridades fijados en la planeación nacional que, por razón de su competencia, les correspondan y de los resultados de las acciones previstas" (art. 8). Asimismo, los programas de las diversas entidades son indispensables para la propia gestión del gobierno federal. El contenido de la Cuenta Pública federal, por ejemplo, deberá tener vínculos con el programa nacional y los programas sectoriales (art. 6), y el propio seguimiento que deberá hacer la Secretaría de Hacienda y Crédito Público de la gestión gubernamental tendrá en consideración entre otras bases, los objetivos y metas del Plan Nacional de Desarrollo y sus programas (art. 9). En otros términos: la planificación tiene un sentido de rendición de cuentas que se concreta en los informes anuales de las dependencias y en la propia gestión interna del gobierno federal.

c) Tiene fines cognitivos y de evaluación. Además, la elaboración de los planes sectoriales tiene varios objetivos importantes. El primario es el conocimiento del estado que guarda el sector del que son responsables la o las dependencias involucradas, es decir, según establece el artículo 26 bis, debe contener un diagnóstico general sobre los problemas por atender, los objetivos específicos, las estrategias para ejecutar las acciones, las líneas de acción que apoyen la implementación de las acciones y los "indicadores estratégicos que permitan dar seguimiento al logro de los objetivos". Por lo tanto, el plan tiene un sentido de conocimiento, priorización de problemas y acciones y evaluación.

Las características de la planeación dan sentido a las políticas públicas que diseña el gobierno en un constante ir y venir de arriba abajo y viceversa, y suponen una actitud distanciada de las concepciones ideológicas del gobernante en turno. Las políticas públicas son un instrumento de gobierno y el juicio que se hace de ellas deviene de la calidad de sus diagnósticos y programas y de la evaluación de los resultados; remiten a los procesos de decisión (Lasswell, 1971: 1), a decisiones y acciones del régimen político frente a situaciones socialmente problemáticas (Salazar Vargas, 2012: 49) o, vistas desde una perspectiva integral o sistémica, a un conjunto de acciones intencionales y causales definidas en la interlocución de gobierno y ciudadanía públicas y legítimas y que configuran un patrón de comportamiento del gobierno y la sociedad (Aguilar Villanueva, 2010: 29). También conllevan un cambio de enfoque en el sentido de que las definiciones ideológicas prescindían de la problematización de las situaciones concretas en favor de respuestas seguras, apriorísticas, que recusaban con facilidad la realidad concreta. Hoy es posible reconocer los límites de ese planteamiento. Las relaciones de poder efectivamente influyen en la conformación de las políticas, pero la autonomización de los diversos campos de ejercicio de la actividad pública, la pluralidad y diferenciación social, la descentralización, la cada vez mayor relevancia de los poderes locales y la paulatina aceptación de formas legítimas a las cuales cualquier gobierno debe sujetarse en la elaboración de una determinada política hacen que cada área de acción pública deba ser analizada en su propia especificidad y dinámica.

Así, una política pública en el campo de la cultura debe partir naturalmente del agente legítimo capacitado para la toma de una decisión, el cual se ciñe a mecanismos establecidos para ello en las leyes y acuerda acciones de carácter vinculante para los diversos agentes sociales implicados en la definición de tal

5 Con anterioridad, Malraux había insistido en incorporar el Ministerio de Cultura al IV Plan de Desarrollo Económico y Social (1962-1965), pues de ese modo figuraría en los presupuestos anuales del Estado. 
política. Supone por esto tanto al agente institucional que toma una decisión y a la decisión misma como al seguimiento del curso de acciones que se derivan de la propia toma de decisión y la vigilancia de los efectos que de manera directa o indirecta afectan su campo de actividad o cualquier otro.

\section{Los programas culturales}

Como he señalado, a partir de la creación del Conaculta dio inicio la elaboración de programas específicos para atender el desarrollo del sector cultural. Antes de la existencia del Conaculta los programas culturales se presentaban como un apartado de los planes de la Secretaría de Educación Pública. Así, por ejemplo, para el periodo 1977-1982, la cultura era tratada en un apartado en el que se hacía mención del "patrimonio, las bellas artes, las culturas populares, la cultura impresa, la cultura y la educación audiovisual, las asociaciones culturales y la protección de la obra artística e intelectual y los intercambios”. Las líneas generales de política cultural se sostenían en múltiples concepciones del mundo, donde "el Estado debe estar al servicio de la cultura y ésta al de la mayoría" y la tarea del poder público era impulsar una cultura crítica y democrática y establecer un puente entre ésta y la educación" (Martínez, 1977: 67). En el periodo 1983-1988, el Plan Nacional de Desarrollo trató de la cultura en un apartado de "Educación, cultura, recreación y deporte", en el que hablaba del desarrollo de una cultura nacional. En el programa nacional respectivo se hacían algunas puntualizaciones sobre patrimonio, descentralización, promoción y difusión de la cultura. ${ }^{6}$

"La creación del Consejo Nacional para la Cultura y las Artes en 1988 representó un punto de inflexión para la política y la gestión cultural en México”, dice Alfonso Castellanos Ribot (2014: 233) y con él muchos más observadores de las políticas públicas de cultura en el país. Sin embargo, existía una situación ambigua, pues el consejo era una institución creada para coordinar las instituciones culturales, pero dependía orgánicamente del Secretario de Educación Pública, por lo tanto, los planes expresaban cierta autonomía, mas no podían elaborarse sin la anuencia del titular del sector educativo.

¿Qué peculiaridades han tenido los planes sectoriales de cultura? De 1989 a 2018 se publicaron cinco programas sectoriales de cultura que abarcan los gobiernos de Carlos Salinas de Gortari (Cs) y Ernesto Zedillo Ponce de León (Ez) del PRI, Vicente Fox (VF) y Felipe Calderón (FC) del Partido Acción Nacional, PAN y Enrique Peña Nieto (EPN) nuevamente del PRI. En ellos predominan los siguientes aspectos.

- Prioritario papel del patrimonio en la política de cultura. Salvo el programa de 2013-2018 (EPN), la preservación, difusión e investigación del patrimonio fue el primer tema mencionado. Rafael Tovar y de Teresa, presidente del Conaculta de 1992-2000 y luego de 2012 a 2016 (donde incluyo su designación como primer Secretario de Cultura), afirmó en un balance de la política cultural de los años noventa que el patrimonio era "el buque insignia de la política cultural" (1994). El programa de Peña Nieto no eliminó la gran relevancia del patrimonio, pero decidió colocarlo en cuarto sitio sin ofrecer alguna explicación sobre este orden.

- Aliento a la creatividad artística. Al poco tiempo de la creación del Consejo Nacional para la Cultura y las Artes se constituyó el Fondo Nacional para la Cultura y las Artes (Fonca). Este organismo es el principal instrumento de apoyo a artistas y creadores y a la promoción de nuevos campos de desarrollo del arte. Ha sido objeto de diversas polémicas durante toda su existencia, una de las más frecuentes es su parcialidad, aunque ha habido otras más serias y constructivas. Estas críticas casi nunca implicaban proponer su desaparición, sino hasta tiempos muy recientes. La estabilidad del Fonca se debe a que supuso un cambio radical en el modo de expresión del compromiso público con la cultura, al asumir que el juicio de los pares era el único criterio para el otorgamiento de los recursos en apoyo a los artistas. El modelo del Fonca sirvió además para encauzar recursos a las culturas populares y ha favorecido la concepción de fondos estatales y fondos mixtos de apoyo a la creatividad.

- Descentralización. Durante los cinco primeros planes de cultura se puso un gran énfasis en la descentralización del aparato cultural a partir de la promoción y el apoyo de iniciativas culturales de los estados y municipios. En un principio este proceso se hizo de un modo imitativo, al replicar con escasa originalidad el modelo del Conaculta. ${ }^{7}$

6 Diario Oficial de la Federación, 31 de mayo de 1983, apartado 7.2.

7 Hubo institutos estatales de cultura desde antes de 1989, como en Guerrero (1983), Aguascalientes (1985) o Campeche (1985). Pero fue después de ese año cuando el proceso de institucionalización cultural en los estados fue más notable. 
Sin embargo, es cierto también que las entidades federativas desarrollaron con profusión sus propias instituciones culturales y las normatividades respectivas, dando lugar a procesos atractivos y avanzados en diversas materias como el registro de creadores en los estados o el impulso de procesos innovadores de difusión o educación artística.

- Innovación. En los diversos planes de cultura puede advertirse un esfuerzo de innovación ante la emergencia de problemas o situaciones desconocidas en el campo de la cultura. Por ejemplo, en el segundo programa (1995-2000, de Ez) se planteó como un objetivo la descentralización de los bienes y servicios culturales y la cooperación cultural internacional. Asimismo se erigieron dos importantes programas especiales de desarrollo cultural infantil y desarrollo cultural de los trabajadores (redefinido luego como programa de animación cultural). El siguiente programa (2001-2006, de vF) transformó el programa de descentralización en vinculación cultural y ciudadanización. El de 2007-2012 (de FC), creó sendos programas específicos sobre infraestructura cultural e industrias culturales y el de 2013-2018 (de EPN) innovó proponiendo un programa para "Posibilitar el acceso universal a la cultura aprovechando los recursos de la tecnología digital", lo que luego derivó en la agenda digital de cultura que propuso el gobierno mexicano en la Cumbre Iberoamericana de 2014.

- Escasa participación. En cuanto a la elaboración de los programas, éstos han adolecido de una pobre participación ciudadana. No ha habido interés o capacidad para promover la amplia participación de la sociedad en la construcción de los programas ni en la discusión de las formas de lograr los objetivos propuestos. La elaboración, en cambio, ha dependido de una burocracia cultural consistente y capaz, que permaneció en el sector cultural por largo tiempo, a pesar de los cambios en las administraciones públicas.

- Falta de presupuestación y de criterios de evaluación. Una crítica común a todos los programas culturales elaborados por los diversos gobiernos desde 1989 es la ausencia de una presupuestación precisa y de elementos claros para la evaluación de los programas. Esto ha generado gran desconfianza hacia la planeación cultural y los ha convertido en pobres instrumentos para la corrección de las políticas públicas de cultura. Salvo en muy escasos momentos, los programas han ofrecido criterios de evaluación y nunca se ha partido de ésta para formalizar un nuevo programa de trabajo.

En una visión de conjunto (cuadro 1) puede observarse la continuidad y los cambios o problemas emergentes que los diversos planes de cultura han tenido desde 1989 hasta 2018, año de terminación del gobierno de Peña Nieto, quien en 2015 propuso convertir el Conaculta en Secretaría de Cultura (sc). Este cambio, desde el punto de vista de la ley de planeación, transformó los programas de cultura de especiales en sectoriales, es decir, al ser Conaculta un órgano subordinado a la Secretaría de Educación Pública su capacidad de planeación dependía de este organismo (por eso se presentaba deespués del programa de educación). Ahora, como secretaría de Estado, le corresponde elaborar un programa sectorial.

\section{La planeación cultural a inicios del gobierno de López Obrador}

El 3 de julio de 2020 se publicó en el Diario Oficial de la Federación el Programa Sectorial Derivado del Plan Nacional de Desarrollo 2020-2024. Una gran incertidumbre rodeó su publicación porque la Secretaría de

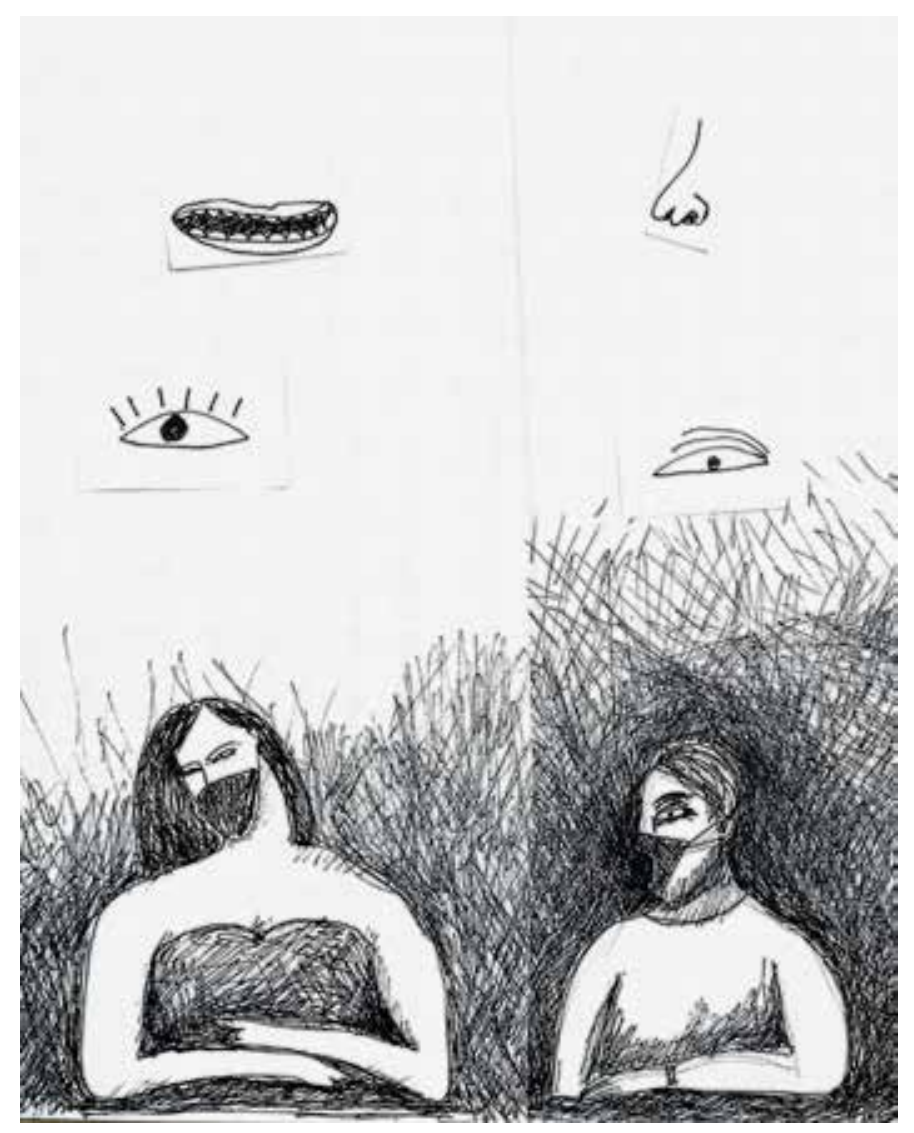




\section{Cuadro 1}

\section{Contenido de los programas sustantivos de cultura en México, 1989-2018}

\begin{tabular}{|c|c|c|c|c|}
\hline 1989-1994 & $1995-2000$ & 2001-2006 & 2007-2012 & 2014-2018 \\
\hline $\begin{array}{l}\text { 1. Preservación y difusión } \\
\text { del patrimonio cultural } \\
\text { nacional } \\
\text { 2. Aliento a la creatividad } \\
\text { artística y a la difusión de } \\
\text { las artes } \\
\text { 3. Desarrollo de la educa- } \\
\text { ción y de la investigación } \\
\text { en el campo de la cultura } \\
\text { y las artes } \\
\text { 4. Fomento del libro y la } \\
\text { lectura } \\
\text { 5. Preservación y difusión } \\
\text { de las culturas populares } \\
\text { 6. Fomento y difusión de } \\
\text { la cultura a través de los } \\
\text { medios audiovisuales de } \\
\text { comunicación }\end{array}$ & $\begin{array}{l}\text { 1. Preservación, inves- } \\
\text { tigación y difusión del } \\
\text { patrimonio cultural } \\
\text { 2. Educación e investiga- } \\
\text { ción artísticas } \\
\text { 3. Difusión de la cultura } \\
\text { 4. Cultura en medios } \\
\text { audiovisuales } \\
\text { 5. Fomento del libro y la } \\
\text { lectura } \\
\text { 6. Estímulo a la creación } \\
\text { artística } \\
\text { 7. Fortalecimiento y } \\
\text { difusión de las culturas } \\
\text { populares } \\
\text { 8. Descentralización de } \\
\text { los bienes y servicios } \\
\text { culturales } \\
\text { 9. Cooperación cultural } \\
\text { internacional }\end{array}$ & $\begin{array}{l}\text { Campos de acción prin- } \\
\text { cipales: } \\
\text { 1. Investigación y con- } \\
\text { servación del patrimonio } \\
\text { cultural } \\
\text { 2. Culturas populares e } \\
\text { indígenas } \\
\text { 3. Patrimonio, desarrollo } \\
\text { y turismo } \\
\text { 4. Estímulo a la creación } \\
\text { artística } \\
\text { 5. Educación e investiga- } \\
\text { ción en el campo artístico } \\
\text { y cultural } \\
\text { 6. Difusión cultural } \\
\text { 7. Lectura y libro } \\
\text { 8. Medios audiovisuales. } \\
\text { 9. Vinculación cultural y } \\
\text { ciudadanización } \\
\text { 10. Cooperación interna- } \\
\text { cional }\end{array}$ & $\begin{array}{l}\text { Ejes de la política cul- } \\
\text { tural: } \\
\text { 1. Patrimonio y diversi- } \\
\text { dad cultural } \\
\text { 2. Infraestructura cul- } \\
\text { tural } \\
\text { 3. Promoción cultural } \\
\text { nacional e internacional } \\
\text { 4. Estímulos públicos a la } \\
\text { creación y mecenazgo } \\
\text { 5. Formación e investiga- } \\
\text { ción antropológica, histó- } \\
\text { rica, cultural y artística } \\
\text { 6. Esparcimiento cultural } \\
\text { y fomento de la lectura } \\
\text { 7. Cultura y turismo } \\
\text { 8. Industrias culturales }\end{array}$ & $\begin{array}{l}\text { Objetivos: } \\
\text { 1. Promover y difundir } \\
\text { las expresiones artísticas } \\
\text { y culturales de México, } \\
\text { así como proyectar la } \\
\text { presencia del país en el } \\
\text { extranjero } \\
\text { 2. Impulsar la educación } \\
\text { y la investigación } \\
\text { artística y cultural } \\
\text { 3. Dotar a la infraestruc- } \\
\text { tura cultural de espacios } \\
\text { y servicios dignos y hacer } \\
\text { un uso más intensivo de } \\
\text { ella } \\
\text { 4. Preservar, promover } \\
\text { y difundir el patrimonio y } \\
\text { la diversidad cultural } \\
\text { 5. Apoyar la creación } \\
\text { artística y desarrollar las } \\
\text { industrias creativas para } \\
\text { reforzar la generación y } \\
\text { acceso de bienes y servi- } \\
\text { cios culturales } \\
\text { 6. Posibilitar el acceso } \\
\text { universal a la cultu- } \\
\text { ra aprovechando los } \\
\text { recursos de la tecnología } \\
\text { digital }\end{array}$ \\
\hline
\end{tabular}

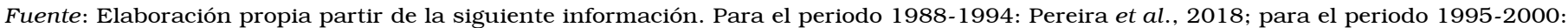
Conaculta, 2000: 15-24; para el periodo 2001-2006: Conaculta, 2001; para el periodo 2007-2012: Conaculta, 2007; para el periodo 2014-2018: Conaculta, 2014.

Cultura dio pocos elementos sobre su formulación y sí en cambio abrió la puerta a muchas especulaciones.

El Programa contiene un diagnóstico, presenta las condiciones normativas en que se desenvuelve la acción cultural del Estado, define principios de acción, presenta seis objetivos básicos de los que deriva 27 estrategias prioritarias y 131 acciones e incluso propone 18 parámetros para juzgar o evaluar el plan. Desde el punto de vista técnico, el plan supone un esfuerzo por generar un instrumento guía de la acción cultural que se ajusta en general a los criterios de planeación de las políticas públicas e incluso innova en la formulación de los parámetros de evaluación mencionados.

Dos tipos de críticas pueden formularse en relación con el documento. Una, que llamo externa, nace de colocar el plan a contraluz de la situación actual de la acción pública de cultura. Por ejemplo, las políticas de apoyo a la creación que define el programa son confrontadas con los presupuestos, la desaparición del Fonca y el intento de suprimir el Fondo de Inversión y
Estímulos al Cine, Fidecine. Lo mismo puede decirse de la pretensión de descentralizar la infraestructura y la oferta cultural ("redistribución de la riqueza cultural") a pesar de la muy notable centralización del presupuesto de la Secretaría de Cultura. Otro tipo de crítica sería interna y consiste en tratar de mirar el programa como un producto de la planificación, es decir, como un documento técnico, y valorar sus cualidades y alcances. Aunque es difícil separar la crítica externa de la interna, por el momento me centro en esta última perspectiva para señalar algunas particularidades del programa.

Las propuestas de política pública en materia de cultura del gobierno de López Obrador han seguido una secuencia coherente desde sus primeras formulaciones, aunque hay una diferencia entre las propuestas elaboradas por su partido y las del equipo responsable del área de cultura. Estas diferencias se observan en la mayor precisión de las últimas. Seguiremos en orden las distintas formulaciones en esta materia. 


\section{Las propuestas culturales en el programa electoral de AMLO}

Con el nombre de Proyecto de nación 2018-2024, el programa del partido Movimiento de Regeneración Nacional, Morena, dibujó a grandes rasgos las líneas de su proyecto. En general se trata de un documento que mezcla diagnóstico, análisis y propuestas, algunas de ellas muy mal elaboradas y otras sorprendentes por ser ajenas al pensamiento de izquierda o ser copia de otros proyectos. ${ }^{8}$ En cuanto a la cultura, lo definido en ese documento de 460 páginas sólo abarcaba un punto con el título de "Cultura comunitaria" (pp. 444446). El documento se centra más en la situación del sector cultural, sobre todo en "el profundo desconocimiento del país” de quienes han diseñado las políticas públicas por carecer de "una visión de tierra, de [una] visión de país como un todo complejo y vital" (Morena, 2018: 444). Señala también que "Los programas base de la Secretaría de Cultura Federal y de los gobiernos estatales, no tienen una política social dentro de su actividad, tienen poca relación con las personas que crean y viven la cultura" (Morena, 2018: 444).

No obstante, ese documento hace escasas propuestas en materia de política pública de cultura. Sólo concluye: "Basados en experiencias concretas y probadas se propone el más importante programa de Cultura Comunitaria que haya implementado el Estado en México. Se reconocerá la fuerza de la cultura como imprescindible en los procesos de paz, de reconstrucción de comunidad, concordia y armonía de las comunidades" (Morena, 2018: 446). En contraparte, todos los rubros tradicionales de la política cultural como el patrimonio, el apoyo a la creatividad y la difusión, la educación artística o la cooperación internacional son marginados, porque usualmente carecían de una política social vinculada a las comunidades.

\section{Las propuestas}

de la futura secretaria de Cultura, Alejandra Frausto

Quizá por lo precario de este proyecto, la futura secretaria de Cultura difundió en junio de 2018, a pocos días de las elecciones, un pequeño folleto que tituló $\mathrm{El}$ poder de la cultura, ${ }^{9}$ donde define con mayor detalle los principios y líneas de sus programas. Éstos son:
- Redistribución de la riqueza cultural (por redistribución entiende el impulso a las culturas locales y sus creaciones), que abarca cuatro programas: Misiones culturales, Patrimonio, Patrimonio cultural inmaterial y Circuitos culturales.

- Cultura para la paz y la convivencia, con los programas: Acercamiento a las artes desde la primera infancia, Vida a la infraestructura cultural, Polos culturales comunitarios y Recuperación afectiva del espacio público.

- Economía cultural, con los siguientes programas: Industrias culturales y empresas creativas, Participación privada, Derechos de autor y derechos de propiedad intelectual comunitaria y Financiamiento cultural.

- Agenda digital, que incluye seis programas: Distribución, Acceso, Fortalecimiento de los derechos de autor, Apropiación tecnológica, Una nueva agenda digital y Cultura científica y arte.

- Jóvenes en la cultura, con los programas de: Apropiación cultural y digital, Fortalecimiento de artes y oficio, Narrativas digitales, Innovación social e Inserción en la economía cultural.

- Vida creativa, con cuatro programas: Enseñanza del arte, Investigación y profesionalización en arte, estudios de la cultura, promoción y gestión cultural, Enfoque comunitario y Museos vivos.

Además, señalaba los siguientes temas prioritarios: Perspectiva de género, Mirar de frente al mundo; De la cultura del poder al poder de la cultura (que se refiere exclusivamente al acondicionamiento de la residencia oficial "Los Pinos" como centro cultural, pues ya no la usaría AMLO) y Patrimonio dañado por los sismos (de 2017).

\section{Las propuestas del Plan Nacional de Desarrollo}

Por disposición legal, el nuevo gobierno debía someter al Congreso de mayoría morenista el Plan Nacional de Desarrollo PND 2019-2024. La ley dispone que es el secretario de Hacienda y Crédito Público el responsable de la elaboración del PND y así lo realizó Carlos Urzúa, quien ostentó el cargo hasta julio de 2019, es decir casi siete meses. El plan elaborado por Urzúa siguió las pautas de la normatividad vigente y dedicó a cultura

8 https://contralacorrupcion.mx/trenmaya/assets/plan-nacion.pdf.

9 La versión "amplia" del documento está en: https: / /es.scribd.com/document/390892591 / EL-PODER-DE-LA-CULTURApdf. Una edición gráfica que alcanzó mayor difusión está en https: / / www.elfinanciero.com.mx/elecciones-2018/el-poderde-la-cultura-nuevo-documento-de-politicas-de-amlo. La diferencia entre uno y otro documento es que el primero tiene una introducción de Alejandra Frausto un poco más extensa. 
el apartado 2.9 del capítulo "Bienestar" (páginas 118 122). El apartado inicia recordando el fundamento legal de la intervención del Estado en materia de cultura e insiste en que se trata de un derecho humano. Resalta la diversidad cultural, recoge algunos datos sobre la infraestructura y el patrimonio material e inmaterial, la planeación artística y se centra en el tema del acceso y la inclusión. Define siete estrategias:

2.9.1 Fomentar el acceso a la cultura de toda la población, promoviendo la redistribución de la riqueza cultural y desarrollando esquemas de planeación intercultural inclusiva y participativa.

2.9.2 Impulsar la formación y profesionalización artística y cultural de los individuos, comunidades, colectivos y trabajadores de la cultura, y brindar opciones de iniciación, capacitación y actualización para toda la población.

2.9.3 Promover y ampliar la oferta cultural a lo largo del territorio nacional y desarrollar el intercambio cultural de México con el extranjero.

2.9.4 Salvaguardar y difundir la riqueza patrimonial de México, tanto material como inmaterial, así como promover la apropiación social de las humanidades, las ciencias y las tecnologías.

2.9.5 Fortalecer las industrias culturales y empresas creativas para generar y difundir sus contenidos.

2.9.6 Desarrollar y optimizar el uso de la infraestructura cultural pública, atendiendo las particularidades y necesidades regionales del país.

2.9.7 Reconocer, preservar, proteger y estimular la diversidad cultural y lingüística de México, con particular atención a los aportes de los pueblos indígenas y afromexicano y otros grupos históricamente discriminados [Gobierno de la República, 2019: 121-122]. ${ }^{10}$

Finalmente, el PND elaborado por Carlos Urzúa no fue del agrado del presidente López Obrador. Lo percibió como "continuismo. Era una concepción todavía en la inercia neoliberal y había que marcar la diferencia". ${ }^{11}$ Un año después, el presidente todavía describía esta situación de una forma que dejaba ver la audacia, pero también la precariedad del documento que sustituyó al que elaboró el primer secretario de Hacienda y Crédito Público: "sentí, cuando me lo presentaron, que era más de lo mismo, como si lo hubiese hecho Aspe o Gil Díaz, con todo respeto, o Carstens, hasta ahí me quedo". "Entonces, afortunadamente teníamos tiempo y en una semana se escribió el nuevo, el que se entregó al Congreso y se desechó el otro". ${ }^{12}$ Es decir, no se distinguía de los planes de la "época neoliberal", al grado de usar hasta el mismo lenguaje técnico. Por ello decidió a toda prisa elaborar otro documento que mostrara la ruptura que representaba su gobierno. Ese otro documento, dijo, "tiene como contexto el plan liberal de 1906 y el plan sexenal del general Lázaro Cárdenas [1934], sin términos propios de la política neoliberal". ${ }^{13}$ Así, resultó un texto básicamente declarativo, una suma de sus promesas de campaña con escasa exactitud de sus alcances. Someter al Congreso dos planes generó confusión, pues a algunos diputados les pareció que el Ejecutivo no tenía precisas sus metas ni se entendía la relación entre uno y otro documento. Esta situación fue "solventada" indicando que el verdadero Plan Nacional era el elaborado por el presidente y que el realizado por el secretario de Hacienda y Crédito Público sólo era un anexo. Al final, sólo se aprobó el primer documento que fue publicado en el Diario Oficial de la Federación el 12 de julio de 2019, es decir, fue "desechado" el plan de Urzúa.

Se trata de un documento hecho con demasiada prisa y mínima supervisión a partir de los discursos del propio presidente. Hay temas tan relevantes como el ambiental que no está tratado en el documento y otros son referidos de manera incompleta. En materia de cultura la formulación es muy pobre y limitada. Por ejemplo, en los cuatro párrafos que le dedica, se menciona al Instituto Nacional de Bellas Artes y Literatura (INBAL) pero no al Instituto Nacional de Antropología e Historia (INAH). Son cuatro las líneas centrales sobre la cultura:

- Amplio significado de la noción de cultura.

- Sentido no excluyente de la política cultural y reconocimiento de su importancia para la paz, la convivencia y la espiritualidad.

- Priorización de los sectores más marginados.

- Atención a las materias tradicionales que han atendido las instituciones culturales sin que éstas centralicen o monopolicen la actividad cultural.

${ }^{10}$ Disponible en http: / gaceta.diputados.gob.mx/PDF/64/2019/abr/20190430-XVIII- 1.pdf.

${ }^{11}$ https: / /www.jornada.com.mx/ultimas / politica/2019/07/ 11 /amlo-rechace-a-urzua-su-pnd-de-201 cinercia-neoliberal 201d-6070.html.

${ }^{12}$ https://lopezobrador.org.mx/2020/05/07/version-estenografica-de-la-conferencia-de-prensa-matutina-del-presidenteandres-manuel-lopez-obrador-316/.

${ }^{13}$ https: / www.jornada.com.mx/ultimas / politica/2019/07/10/admite-amlo-que-tuvo-discrepancias-con-el-ex-secretariode-hacienda-8772.html. 
Con lo mencionado hasta este momento sobre la política cultural del gobierno de López Obrador se tiene clara la centralidad de la acción comunitaria y, desde luego, la intención de mantener las líneas de la política cultural seguidas en los últimos años: patrimonio, fomento a la cultura, educación artística, industrias culturales (sostenidas en una concepción amplia de los derechos culturales). Con todo, la planificación como tal no se ha hecho, y esto, más la forma en que estos compromisos se materializan en el presupuesto cultural, suscitan algunas reflexiones.

\section{Programa Sectorial}

derivado del Plan Nacional de Desarrollo 2020-2024

Este documento expresa continuidad con los documentos anteriores, pero también marca giros relevantes. Es evidente que ha predominado la visión del equipo dirigente de la Secretaría de Cultura que sostiene expresiones como la ya mencionada "redistribución de la riqueza cultural" o "el poder de la cultura", que en la práctica se refieren a una posible descentralización de la acción cultural y la conversión de la antigua residencia oficial de Los Pinos en un centro cultural, respectivamente.

El tono rupturista de documentos anteriores -a "lo largo de la historia no se ha registrado ninguna transformación social que no haya sido acompañada de una renovación cultural" $-{ }^{14}$ se atempera en el programa, al tomar como punto de partida el grueso de la acción cultural del Estado mexicano y marcar sendas de notable continuidad. Por ejemplo, en relación con la educación artística habla de "consolidar la tarea educativa del sector cultura". Esto se ve con mayor nitidez en el diagnóstico denominado "Análisis del estado actual", donde se presenta información sobre la brecha en el acceso y disfrute de los bienes y servicios culturales, la infraestructura cultural, el sistema de financiamiento y las lenguas indígenas, entre otros. Esta información es analizada a partir del prisma de valores que enmarcan las definiciones pasadas y actuales del país en materia de cultura: derechos culturales, la aceptación entusiasta de la diversidad y la defensa de ésta y la decisión de luchar contra la exclusión y el entorno de violencia en que viven las comunidades pobres. Es notable que el concepto cultura comunitaria sólo aparezca en el anexo: "Términos

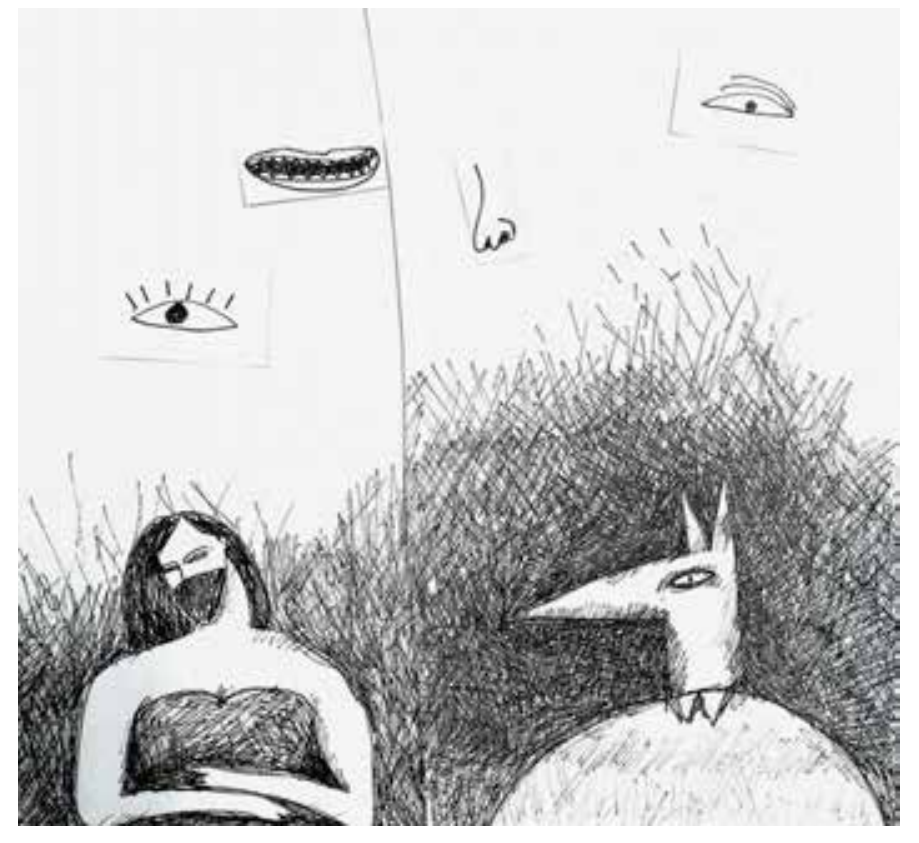

comunes utilizados por organismos y áreas del Sector Cultura", cuando fue presentado como el núcleo de la política pública de este sexenio en materia de cultura.

Los objetivos del programa son los siguientes:

1. Reducir la desigualdad en el ejercicio de los derechos culturales de personas y comunidades, prioritariamente en contextos de vulnerabilidad, con su participación en procesos que fortalezcan los ciclos, prácticas e identidades culturales [tres estrategias, siete acciones prioritarias, tres parámetros de evaluación].

2. Consolidar la tarea educativa del Sector Cultura para brindar a las personas mejores opciones de formación, actualización y profesionalización, en todos sus niveles y modalidades, bajo criterios de inclusión y reconocimiento de la diversidad [cuatro estrategias, 23 acciones prioritarias, tres parámetros de evaluación].

3. Garantizar progresivamente el acceso a los bienes y servicios culturales a las personas, a través del incremento y diversificación de la oferta cultural en el territorio y del intercambio cultural de México con el extranjero [seis estrategias, 28 acciones prioritarias, tres parámetros de evaluación].

4. Proteger y conservar la diversidad, la memoria y los patrimonios culturales de México mediante acciones de preservación, investigación, protección, promoción de su conocimiento y apropiación [cuatro estrategias, 29 acciones prioritarias, tres parámetros de evaluación].

${ }^{14}$ Son las primeras palabras del documento El poder de la cultura, de Alejandra Frausto. 
5. Fortalecer la participación de la cultura en la economía nacional a través del estímulo y profesionalización de las industrias culturales y empresas creativas, así como de la protección de los derechos de autor [cinco estrategias, 25 acciones prioritarias, tres parámetros de evaluación].

6. Enriquecer la diversidad de las expresiones creativas y culturales de México mediante el reconocimiento y apoyo a los creadores, académicos, comunidades y colectivos [tres estrategias, 13 acciones prioritarias, tres parámetros de evaluación] [Secretaría de Cultura, 2020].

Puede observarse que los temas relacionados con educación artística, acceso a los bienes y servicios culturales, memoria y patrimonio y economía cultural dan lugar al mayor número de estrategias (21) y acciones prioritarias (105). El primer objetivo, relativo a la reducción a la desigualdad y el último referido al enriquecimiento de diversidad de las expresiones creativas y culturales remiten a seis estrategias y 26 acciones prioritarias (cuadro 2).

\section{Crisis de la política pública de cultura}

Crisis nos remite a una situación grave y decisiva que pone en peligro el desarrollo de un asunto o de un proceso. Etimológicamente implica separar o juzgar, pero también indica ruptura, daño o inestabilidad. Supone tanto una situación como el ejercicio de un juicio sobre ésta. Al analizar la política pública de cultura me he centrado en la planeación realizada a partir de 1990, porque representó el cambio más visible del nuevo modelo institucional de cultura. Esto trajo adosada la definición de campos de actuación, establecimiento de prioridades, reconocimiento de nuevos espacios y problemas de intervención y búsqueda de

\section{Cuadro 2}

Definiciones del programa de cultura de Andrés Manuel López Obrador 2019-2024

\begin{tabular}{|c|c|c|c|c|}
\hline $\begin{array}{c}\text { Definiciones del } \\
\text { Programa del partido } \\
\text { Morena } \\
\text { (Proyecto de nación) } \\
2017\end{array}$ & $\begin{array}{c}\text { Definiciones de } \\
\text { Alejandra Frausto, } \\
\text { anunciada } \\
\text { secretaria de Cultura } \\
\text { (El poder de la cultura) } \\
\text { Junio } 2018\end{array}$ & $\begin{array}{c}\text { Definiciones del } \\
\text { Proyecto de Plan } \\
\text { Nacional de Desarrollo } \\
\text { (PND-Suplemento), del } \\
\text { secretario de Hacienda } \\
\text { y Crédito Público } \\
\text { Abril } 2019\end{array}$ & $\begin{array}{c}\text { Definiciones del } \\
\text { Proyecto de Plan } \\
\text { Nacional } \\
\text { (PND) de Andrés Manuel } \\
\text { López Obrador } \\
\text { Abril } 2019\end{array}$ & $\begin{array}{c}\text { Definiciones del } \\
\text { Programa Sectorial } \\
\text { Derivado del Plan } \\
\text { Nacional de } \\
\text { Desarrollo 2020-2024 } \\
\text { Julio } 2020\end{array}$ \\
\hline $\begin{array}{l}\text { El programa de Cultura } \\
\text { Comunitaria promoverá } \\
\text { el acceso a la cultura } \\
\text { de manera gratuita y el } \\
\text { ejercicio de los derechos } \\
\text { culturales de la población } \\
\text { en situación vulnerable, } \\
\text { con pleno respeto a la } \\
\text { diversidad cultural }\end{array}$ & $\begin{array}{l}\text { 1. Redistribución de la } \\
\text { riqueza cultural } \\
\text { 2. Cultura para la paz y } \\
\text { la convivencia } \\
\text { 3. Economía cultural } \\
\text { 4. Agenda digital } \\
\text { 5. Jóvenes en la cultura } \\
\text { 6. Vida creativa } \\
\text { Temas prioritarios: } \\
\text { - Perspectiva de género } \\
\text { - Mirar de frente al } \\
\text { mundo } \\
\text { - De la cultura del poder } \\
\text { al poder de la cultura } \\
\text { (que se refiere exclusi- } \\
\text { vamente al acondicio- } \\
\text { namiento como centro } \\
\text { cultural de la residencia } \\
\text { oficial "Los Pinos", de } \\
\text { la que ya no haría uso } \\
\text { AmLo) y } \\
\text { - Patrimonio dañado por } \\
\text { los sismos (de 2017) }\end{array}$ & $\begin{array}{l}\text { 1. Fomentar el acceso } \\
\text { a la cultura de toda la } \\
\text { población } \\
\text { 2. Impulsar la forma- } \\
\text { ción y profesionalización } \\
\text { artística y cultural de los } \\
\text { individuos, comunidades, } \\
\text { colectivos y trabajadores } \\
\text { de la cultura } \\
\text { 3. Promover y ampliar la } \\
\text { oferta cultural } \\
\text { 4. Salvaguardar y difun- } \\
\text { dir la riqueza patrimonial } \\
\text { de México, tanto material } \\
\text { como inmaterial } \\
\text { 5. Fortalecer las indus- } \\
\text { trias culturales y empre- } \\
\text { sas creativas } \\
\text { 6. Desarrollar y optimizar } \\
\text { el uso de la infraestructu- } \\
\text { ra cultural pública } \\
\text { 7. Reconocer, preservar, } \\
\text { proteger y estimular la } \\
\text { diversidad cultural y } \\
\text { lingüística de México }\end{array}$ & $\begin{array}{l}\text { 1. Amplio significado de } \\
\text { la noción de cultura } \\
\text { 2. Sentido no excluyente } \\
\text { de la política cultural y } \\
\text { reconocimiento de su } \\
\text { importancia para la paz, } \\
\text { la convivencia y la espiri- } \\
\text { tualidad } \\
\text { 3. Priorización de los sec- } \\
\text { tores más marginados } \\
\text { 4. Atención a las materias } \\
\text { tradicionales que han } \\
\text { atendido las institucio- } \\
\text { nes culturales sin hacer } \\
\text { que éstas centralicen o } \\
\text { monopolicen la actividad } \\
\text { cultural }\end{array}$ & $\begin{array}{l}\text { 1. Reducción de la des- } \\
\text { igualdad en el campo de } \\
\text { la cultura } \\
\text { 2. Consolidación de la } \\
\text { tarea educativa del sector } \\
\text { cultura } \\
\text { 3. Garantizar el acceso } \\
\text { a los bienes y servicios } \\
\text { culturales } \\
\text { 4. Protección y la conser- } \\
\text { vación de la diversidad } \\
5 \text {. Fortalecimiento de } \\
\text { la participación de la } \\
\text { cultura en la economía } \\
\text { nacional y } \\
6 \text {. Enriquecimiento de las } \\
\text { expresiones creativas y } \\
\text { culturales }\end{array}$ \\
\hline
\end{tabular}

Fuente: Elaboración propia. 
alternativas de solución de esos problemas a través de la descentralización, el establecimiento de fondos para el fomento cultural o iniciativas institucionales. ¿Por qué considero que, pese a este activismo, hemos desembocado en una crisis de las políticas públicas de cultura?

\section{La presupuestación cultural}

Uno de los puntos básicos para observar la consistencia de una política pública es la asignación presupuestal. Toda política pública se expresa finalmente en dinero, pero en cultura -aunque también en otros campos-, la planeación se ha realizado sin llegar a la presupuestación. A lo más se ha hecho mención, por ejemplo, de que la totalidad de las acciones que se consideran en el Programa Sectorial de Cultura 2020-2024 y las labores de coordinación interinstitucional "se realizarán con cargo al presupuesto autorizado" (apartado 3), una expresión casi redundante. Esta forma de planeación sin definir recursos abre la posibilidad de ofrecer grandes proyectos llenos de incertidumbre, como ha ocurrido a lo largo de estas tres décadas. En el cuadro 3 puede verse que históricamente ha habido magros presupuestos de cultura, pero en este sexenio la participación del sector cultural en el presupuesto total de la federación es más baja aún. Por otra parte, se cambió la forma de estructurar el presupuesto de cultura, que ha reconfigurado por completo el papel de las instituciones culturales.

El primer presupuesto del gobierno de López Obrador fue más o menos el mismo que se envió desde el ejecutivo y procuró atender las líneas generales definidas en la campaña electoral. En el segundo presupuesto (para 2020) se hizo más claro el sentido de la política pública de cultura que se distancia, en parte, de las tendencias anteriores. El presupuesto de 2019 representó 0.21 por ciento del presupuesto federal. El de 2020 supuso el 0.22 por ciento. El crecimiento de un presupuesto a otro fue de 5.3 por ciento, un poco más que la inflación.

Las instituciones más consolidadas tuvieron un trato semejante a otros ejercicios, con sus más y sus menos. Por ejemplo, el INAH y el INBAL crecieron en su presupuesto 4.77 por ciento y 5.69 por ciento respectivamente. El Centro Nacional de las Artes (CNA) y la Dirección General de Bibliotecas, en cambio, bajaron 13 por ciento. Unos organismos no tuvieron tan drástica caída: Canal 11 aumentó 2.6 por ciento y el Fonca 2.6 por ciento, y así perfilaron en general un año tan difícil como el anterior.

\section{Desinstitucionalización}

Ahora bien, las novedades más notables estuvieron en la gestión misma de la secretaría. El presupuesto se concentró para su ejecución, lo que es un retroceso al decir de algunas voces autorizadas como la de Arturo Saucedo, pues se había luchado mucho en el sentido opuesto. ${ }^{15}$ En 2020 las dos subsecretarías de la institución vieron crecer sus arcas de manera importante. La Subsecretaría de Desarrollo Cultural incrementó su presupuesto casi dos mil por ciento (de 87.14 millones a 1836.2 millones) y la de Diversidad Cultural lo hizo en alrededor de diez mil por ciento (de 6.3 millones a 646 millones). Además de la concentración de los recursos, tras estos incrementos estuvieron dos grandes programas. Uno es Cultura Comunitaria que creció 50 por ciento (pasó de 400 a 600 millones) y otro fue el Complejo Cultural Los Pinos, que se llevó una inversión de 1688 millones, la octava parte del presupuesto de la Secretaría.

Cultura Comunitaria continuará siendo, en lo posible, el programa principal de la secretaría, acorde con las definiciones tomadas desde la campaña y ratificadas por el presidente de la república cuando definió que, para él, "cultura es lo que tiene que ver con los pueblos". ${ }^{16}$ Por cierto, como dice Daniel Giménez Cacho, López Obrador, "por alguna razón no usa la palabra arte, quizás la relaciona con los privilegios burgueses. La cultura y el arte son algo mucho más complejo. Me alarma que un líder tan prominente quiera sólo favorecer su concepción de cultura, tan limitada". ${ }^{17}$ Así, sus proyectos trabajarían en favor de los municipios más pobres manteniendo y ampliando el esfuerzo actual hasta cubrir los 700 municipios de mayor marginalidad y altos índices de violencia.

Este esfuerzo de ninguna manera es desdeñable, pero deben remarcarse sus deficiencias, como que el gobierno federal irrumpa en el ámbito municipal sin el concurso de los gobiernos estatales y el que sea un programa que se autonomiza del resto de los programas culturales dejándolo como un departamento estanco dentro del hacer general de la cultura. Adicionalmente, para la ejecución del programa se mantiene y amplía

\footnotetext{
${ }^{15}$ https: / / www.milenio.com/cultura/presupuesto-para-cultura-2020-resultado-de-la-centralizacion.

${ }^{16} \mathrm{https}$ / / www.eluniversal.com.mx/cultura/lamentan-concepto-de-cultura-de-amlo.

17 https://www.eluniversal.com.mx/cultura/lamentan-concepto-de-cultura-de-amlo.
} 
el esquema laboral de out sourcing, con el que en 2019 se contrataron alrededor de un millar de talleristas y promotores culturales (cuadro 3).

El Complejo Cultural Los Pinos es un proyecto innecesario desde el punto de vista de los requerimientos de la infraestructura cultural de la Ciudad de México y de esa zona de la urbe. Supone por tanto un enorme gasto derivado de una decisión política que, por el gasto que implica, afecta a otras instancias culturales del país. Procede de una decisión caprichosa, sin sustento en ningún diagnóstico y muy alejada de la opinión de muy importantes actores de la comunidad cultural. Se inserta en una idea de "legado" del presidente de la república, como lo fueron el CNA o la Biblioteca Vasconcelos en su momento.

En los hechos, la injerencia del presidente en la planeación cultural ha ido más allá de la autoridad del sector cultura. Casi al mismo tiempo que se dispone que en la administración pública "no se ejercerá el $75 \%$ del presupuesto disponible de las partidas de servicios generales y materiales y suministros", ${ }^{18}$ lo que incluye a la Secretaría de Cultura, se establece la extinción de "todos los fideicomisos públicos sin estructura orgánica, mandatos o análogos de carácter federal" ${ }^{19}$ entre los que se incluyen el Fonca y Fidecine. Por otra parte, el presidente declara como prioritario, es decir, como un programa cuyas acciones y gasto no serán pospuestos, el Espacio Cultural de Los Pinos y Bosque de Chapultepec. ${ }^{20}$ Esta disonancia de los intereses del presidente con respecto de lo que podría ser el sector cultural se manifiesta con el impulso a otros proyectos externos a la Secretaría de Cultura, como el de fomento a la lectura que impulsa el Fondo de Cultura Económica y la Coordinación Nacional Memoria Histórica y Cultural de México, cuyo consejo asesor preside la doctora Beatriz Gutiérrez Müller. ${ }^{21}$ Poco a poco la institucionalidad cultural construida durante tres décadas ha ido quedando marginada frente a las grandes definiciones del poder presidencial.

Es explicable que el presupuesto en época de crisis sufra presiones para resolver otras necesidades, sin embargo, la situación actual del presupuesto del sector cultura revela un problema estructural, en parte heredado y en parte generado por el actual gobierno: a su bajo nivel se añade su reestructuración centralizada, contraviniendo la tendencia decidida desde hace lustros identificada con una idea de democratización de la cultura. El programa más relevante del actual sexenio, Cultura Comunitaria, es un proyecto carente de institucionalidad e ineficaz a la larga, en tanto que se hace sin convenir con los estados en los que se aplica, pues la Secretaría de Cultura carece de la infraestructura para intervenir localmente.

\section{Los objetivos de las políticas públicas de cultura. ¿Metas utópicas?}

En 2018, el profesor Per Mangset de la Universidad de Telemark publicó el artículo "The end of cultural policy?". En éste hace una interesante reflexión sobre las políticas públicas de cultura en las democracias occidentales. Observa que estas últimas no se adaptan a las grandes transformaciones que viven las sociedades contemporáneas, lo que produce desafíos importantes a las políticas culturales. Mangset presenta siete escenarios problemáticos de las políticas culturales: 1) parece ser muy difícil la democratización de la cultura; 2) las autoridades públicas apoyan sistemáticamente instituciones culturales que se pueden considerar obsoletas; 3) los artistas profesionales viven en condiciones de precariedad económica a pesar de los planes de apoyo público; 4) las políticas públicas de cultura son predominantemente nacionales a pesar de la globalización de la producción y distribución cultural; 5) las autoridades públicas justifican el incremento de las subvenciones a la cultura en razón de los efectos benéficos que el arte y la cultura podrían tener más allá del propio campo cultural; no obstante, es posible argumentar que tales efectos podrían ser iguales o mayores si los recursos se aplicaran en otros campos sociales; 6) un sector cultural público específico puede parecer que encarcela la cultura en una jaula de hierro burocrática y, por último, 7) se podría proponer que una política cultural pública no tiene sentido en un periodo de estancamiento de las competencias públicas.

Estos escenarios no están alejados de las condiciones mexicanas: hay enormes déficits de democratización de la cultura en cuanto acceso y participación; muchas instituciones, sobre todo los grandes institutos nacionales de cultura, requieren un rediseño institucional

\footnotetext{
${ }_{18}$ Decreto por el que se establecen las medidas de austeridad que deberán observar las dependencias y entidades de la Administración Pública Federal bajo los criterios que en el mismo se indican (Diario Oficial de la Federación, 23 de abril de 2020 ).

${ }_{19}$ Decreto por el que se ordena la extinción o terminación de los fideicomisos públicos, mandatos públicos y análogos (Diario Oficial de la Federación, 2 de abril de 2020).

${ }^{20}$ Decreto citado del 23 de abril de 2020.

${ }^{21}$ https: / / lopezobrador.org.mx/2019/09/01/discurso-del-lic-andres-manuel-lopez-obrador-presidente-constitucionalde-los-estados-unidos-mexicanos-en-su-primer-informe-de-gobierno/Sobre la presentación.
} 


\section{Cuadro 3}

Presupuesto asignado a las unidades responsables del sector cultura (Ramo 48)

\begin{tabular}{|c|c|c|c|c|c|c|c|c|c|}
\hline & $\begin{array}{c}\text { Inicio } \\
\text { FCH }\end{array}$ & $\begin{array}{c}\text { Inicio } \\
\text { EPN }\end{array}$ & & $\begin{array}{c}\text { Inicio } \\
\text { AMLO }\end{array}$ & & & & & \\
\hline Unidad responsable & $\begin{array}{l}\text { PEF } 2007 \\
\text { (pesos) }\end{array}$ & $\begin{array}{l}\text { PEF } 2013 \\
\text { (pesos) }\end{array}$ & $\begin{array}{l}\text { PEF 2018 } \\
\text { (pesos) }\end{array}$ & $\begin{array}{l}\text { Proyecto PEF } \\
2019\end{array}$ & $\begin{array}{l}\text { Diferencia } \\
\text { 2019-2018 } \\
\text { (pesos) }\end{array}$ & $\begin{array}{l}\text { Diferencia } \\
2019-2018 \\
\text { (\%) }\end{array}$ & $\begin{array}{l}\text { PEF } 2020 \\
\text { (pesos) }\end{array}$ & $\begin{array}{l}\text { Diferencia } \\
2020-2019 \\
\text { (pesos) }\end{array}$ & $\begin{array}{c}\text { Diferencia } \\
2020-2019 \\
\text { (\%) }\end{array}$ \\
\hline DOO INAH & 2064577429 & 3391670377 & 3666582423 & 3739756181 & 71173758 & $1.94 \%$ & 3918082297 & 178326116 & $4.77 \%$ \\
\hline EOO INBAL & 1759060208 & 2885877757 & 3260834371 & 3084290425 & -176543946 & $-5.41 \%$ & 3259871301 & 175580876 & $5.69 \%$ \\
\hline F00 Radio Educación & 50259055 & 97320826 & 85691238 & 76218555 & -9472683 & $-11.05 \%$ & 80952396 & 4733841 & $6.21 \%$ \\
\hline $\begin{array}{l}100 \text { Oficina de la } \\
\text { SE-CUL }\end{array}$ & & & 3805366477 & 4703003680 & 897637203 & $23.59 \%$ & 5266509628 & 563505948 & $11.98 \%$ \\
\hline HOO Conaculta & 3036472423 & 5069162097 & & & & & & & \\
\hline $\begin{array}{l}\text { L3N Centro de } \\
\text { Capacitación } \\
\text { Cinematográfica, A.C. }\end{array}$ & 31930949 & 75671036 & 30368580 & 28709284 & -1659296 & $-5.46 \%$ & 31297080 & 2587796 & $9.01 \%$ \\
\hline $\begin{array}{l}\text { L6U Compañía } \\
\text { Operadora del Centro } \\
\text { Cultural y Turístico de } \\
\text { Tijuana S.A. de C.V. }\end{array}$ & 47171291 & 109677646 & 99958841 & 97865267 & -2093574 & $-2.09 \%$ & 100947175 & 3081908 & $3.15 \%$ \\
\hline $\begin{array}{l}\text { L8G Educal, S.A. } \\
\text { de C.V. }\end{array}$ & 30707506 & 64595939 & 32936736 & 32139534 & -797202 & $-2.42 \%$ & 53202054 & 21062520 & $65.53 \%$ \\
\hline $\begin{array}{l}\text { L8P Estudios } \\
\text { Churubusco Azteca } \\
\text { S.A. }\end{array}$ & 29164285 & 128391358 & 39388807 & 29620195 & -9768612 & $-24.80 \%$ & 29675194 & 54999 & $0.19 \%$ \\
\hline $\begin{array}{l}\text { L9Y Fideicomiso para } \\
\text { la Cineteca Nacional }\end{array}$ & 30493418 & 47366594 & 47216656 & 43669784 & -3546872 & $-7.51 \%$ & 47382573 & 3712789 & $8.50 \%$ \\
\hline MDC Imcine & 210745156 & 374575584 & 274272322 & 231243096 & -43029226 & $-15.69 \%$ & 237782561 & 6539465 & $2.83 \%$ \\
\hline $\begin{array}{l}\text { MHL Televisión } \\
\text { Metropolitana, } \\
\text { S.A. de C.V. }\end{array}$ & 144053133 & 231458278 & 172722910 & 149191510 & -23531400 & $-13.62 \%$ & 155789023 & 6597513 & $4.42 \%$ \\
\hline MDB Inali & & & 85103491 & 74790648 & -10312843 & $-12.12 \%$ & 76319095 & 1528447 & $2.04 \%$ \\
\hline 100 Indautor & & & 75937347 & 70536438 & -5400909 & $-7.11 \%$ & 74855507 & 4319069 & $6.12 \%$ \\
\hline JOO INEHRM & & & 37793783 & 33055662 & -4738121 & $-12.54 \%$ & 34814647 & 1758985 & $5.32 \%$ \\
\hline Fonart & \multicolumn{9}{|c|}{ Aun no aparece en el ramo 48} \\
\hline Total & 7437634853 & 12475767452 & 11716173982 & 12394090259 & 677916277 & $6 \%$ & 13367480531 & 973390272 & $7.85 \%$ \\
\hline $\begin{array}{l}\text { Gasto total federal } \\
\text { (PEF total) } \\
\text { Fuente: Decreto PEF } \\
\text { en el DoF }\end{array}$ & 2160412500000 & 3956361600000 & 5236375600000 & 5814291700000 & & & 6096335800000 & & \\
\hline $\begin{array}{l}\text { Porcentaje sector } \\
\text { Cult/PEF }\end{array}$ & $0.33 \%$ & $0.32 \%$ & $0.22 \%$ & $0.21 \%$ & & & $0.22 \%$ & & \\
\hline
\end{tabular}

Fuente: Los datos hasta 2019 fueron recopilados por Carlos Villaseñor Anaya, quien los ha difundido por diversos medios. Los correspon-

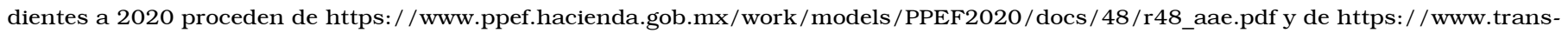
parenciapresupuestaria.gob.mx/es / PTP/infografia_ppef2020

muy difícil de consensuar; los artistas profesionales viven, en su gran mayoría, en condiciones de precariedad; ha habido un enorme esfuerzo por influir y desarrollar las políticas públicas internacionales aunque siempre privilegiando el aspecto nacional; con frecuencia los fondos públicos para la subvención de los procesos culturales han sido cuestionados y casi siempre son muy reducidos; ha habido dificultad para ajustar las políticas públicas de cultura a los requerimientos de la administración, sobre todo las relacionadas con la promoción comunitaria; las políticas públicas de cultura, pese a las muchas declaraciones en sentido contrario, no son prioritarias frente a otras políticas de tipo social o de fomento económico.

Parece paradójico que la definición del sector cultural a favor de las culturas comunitarias implique el deslave de las políticas públicas de cultura vistas como un todo. Como he mostrado a lo largo de este 
texto, la legítima apuesta de la Secretaría de Cultura en favor de las culturas marginadas del campo y la ciudad ha conducido a una cierta escisión de este objetivo respecto de las líneas tradicionales de la política cultural, debido a una planeación deficiente o inconclusa y a un proceso de desinstitucionalización que se expresa también en el cuestionamiento de los fondos públicos de cultura como Fonca y Fidecine y en la desvinculación de la acción de la Secretaría de Cultura de los procesos estatales y locales.

Si Cultura Comunitaria constituye el eje central de la política cultural esto significaría que la política de comunicación, de patrimonio, de formación artística, de cine, radio y televisión culturales y todos los demás aspectos de la política pública de cultura deberían incluirse en Cultura Comunitaria. Mas lo que vemos es la segregación del programa, sin una idea de transversalidad, donde creadores y gestores culturales, y en general todos los agentes sociales interesados en la cultura, trabajen coordinados con ese programa.

\section{Revisión de las políticas públicas de cultura}

El paradigma fundamental de las políticas culturales modernas se centra en la democracia y la diversidad. Esto supone acceso, participación y el despliegue de la creatividad en todas sus formas. Ahora bien, ¿cómo desarrollar una política cultural democrática, incluso a favor de los sectores culturales, sin tomar en cuenta la radio, la televisión, el cine, y ahora el internet y las redes sociales?; ¿es posible escindir la atención a la sociedad en una política preocupada por las mayorías marginadas campesinas e indígenas de otra pendiente de los grupos urbanos populares, medios y altos de la sociedad?

El gobierno federal ha intentado focalizar su acción pública en el desarrollo más que en el crecimiento, pero no ha definido en qué consiste esto. Si se trata de la puesta en marcha de políticas redistributivas que abatan la desigualdad, ¿cómo se expresan en el campo de la cultura?, ¿en la "redistribución de la riqueza cultural", como se enunció en el confuso Proyecto de nación difundido antes de las elecciones? ¿No será mejor considerar que consiste en la ampliación de las capacidades electivas de todos los sectores de la sociedad en materia de cultura?

Una política cultural es inseparable de una idea adecuada de cómo se gestiona. La política define el qué y la gestión el cómo. Para ello se han llevado a cabo diversas reflexiones que han caminado en tres ámbitos: lo que las instituciones deben producir de acuer- do con los fines públicos definidos por las instancias de participación de la sociedad, la definición de estos fines públicos a través de políticas de participación ciudadana, y las medidas de coerción o estímulo para que los objetivos se pongan en práctica. Esto es tanto más necesario para la política pública de cultura, pues no cuenta con los instrumentos para alcanzar a toda la sociedad y exige la participación y el convenio con las instituciones culturales de los estados y los municipios del país. En otras palabras, más que la acción directa en el campo comunitario requiere orquestar la acción comunitaria en todo el país.

Por último, una política cultural necesita instrumentos institucionales que cuenten con normas precisas de operación. En México, los mejor regidos en este aspecto son los institutos, centros y fondos culturales, no así los programas de trabajo comunitarios. Centrar la política cultural en aparatos débilmente definidos institucional y normativamente, como se ha hecho en materia de cultura comunitaria, es abrir un frente de inestabilidad e ineficiencia.

A partir de los criterios que he presentado, considero que hay una crisis profunda de la política cultural en México, agravada por el silencio y la falta de una actitud proactiva de la Secretaría de Cultura federal para construir una visión global de la política cultural en el país. Lo que hoy día ocurre en México sugiere que hay una crisis en la política cultural entendida como política pública. No está en riesgo la institucionalidad cultural en sí misma sino la integralidad de la política de cultura y su vinculación con lo que se ha llamado el sector cultural constituido por el conjunto de actores y empresas culturales que generan una importante parte del producto interno bruto nacional (3.2 por ciento) y da trabajo a más de un millón de personas. La razón de esta desvinculación no está en el enfoque de Cultura Comunitaria, sino en su segregación de la política cultural en su conjunto y en la falta de vínculos que den sentido a toda la orientación cultural del Estado. Una reflexión pública sobre la planeación cultural con el involucramiento de todos los sectores que intervienen en el campo cultural permitiría, quizá, la superación de esta crisis.

\section{Fuentes}

Aguilar Villanueva, Luis F. 1992 La hechura de las políticas, Miguel Ángel Porrúa, México.

Aguilar Villanueva, Luis F.

2010 "Introducción", en Luis F. Aguilar, comp. Política pública, Biblioteca Básica de Administración Pública DF/Siglo xxi Editores, México, pp. 17-60. 
Castellanos Ribot, Alfonso

2014 "Retos de la política cultural en México", en Periférica. Revista para el Análisis de la Cultura y el Territorio, núm. 15, pp. 233-246.

Conaculta

2000 "Nueva proyección de la política cultural", en Memoria 1995-2000, t. I, Consejo Nacional para la Cultura y las Artes, México, pp. 15-24.

Conaculta

2001 Programa Nacional de Cultura 2001-2006. La cultura en tus manos, Consejo Nacional para la Cultura y las Artes, México.

Conaculta 2007

Programa Nacionalde Cultura 2007-2012, Consejo Nacional para la Cultura y las Artes, México <https: / /www.cultura.gob.mx/recursos / acerca_de/pnc2007_20121.pdf>.

Conaculta

2014 Programa Especial de Cultura y Arte (20142018), en Diario Oficial de la Federación, 28 de abril.

LASSWELl, HAROLD D.

1971 A Pre-view of Policy Sciences, American Else-

Mangset, Per vier, Nueva York.

2018 "The end of cultural policy?", en International Journal of Cultural Policy, vol. 26, núm. 3, pp. 398-411, DOI: $10.1080 / 10286632.2018$.

Martínez, Eduardo 1500560

1977 La política cultural de México, Organización de las Naciones Unidas para la Educación, la
Ciencia y la Cultura (Políticas culturales: estudios y documentos), París <https: / / unesdoc. unesco.org/ark: /48223/pf0000037076>.

Morena

2018 Proyecto de nación 2018-2014, Movimiento de Regeneración Nacional <https://contrala corrupcion. $\mathrm{mx} /$ trenmaya / assets / plannacion.pdf $>$.

Pereira, Armando, Claudia

Albarrán, Juan Antonio Rosado

Y ANGÉlicA TORNERO

2018 "Consejo Nacional para la Cultura y las Artes (Conaculta)", en Enciclopedia de la Literatura en México <http: / /www.elem.mx/institucion/ datos /323>.

Ruiz Dueñas, Jorge

1983 "La vía de la planificación mexicana", en $R e$ vista de Administración Pública, núm. 55-56, julio-diciembre, pp. 35-64.

Salazar VARGas, Carlos

2012 "La definición de política pública", en Bien Común, vol. 18, núm. 209, junio-julio, pp. 47-52 <http://www.fundacionpreciado.org. $\mathrm{mx} /$ biencomun/bc209/C_Salazar.pdf>.

Secretaría de Cultura

2020 Programa Sectorial Derivado del Plan Nacional de Desarrollo 2020-2024, en Diario Oficial de la Federación, 3 de julio.

Tovar y de TEREsa, Rafael

1994 Modernización y política cultural, una visión de la modernización de México, Fondo de Cultura Económica, México. 